\title{
Effect of coordination on bond properties: A first principles study
}

\author{
JAITA PAUL and SHOBHANA NARASIMHAN* \\ Theoretical Sciences Unit, Jawaharlal Nehru Centre for Advanced Scientific Research, Bangalore 560 064, India
}

\begin{abstract}
We have used density functional theory to obtain the binding curves for a variety of hypothetical periodic structures of $\mathrm{Al}, \mathrm{Si}, \mathrm{Pb}, \mathrm{Sn}$ and $\mathrm{Au}$. Upon examining the resulting database of results for equilibrium bond lengths and radial force constants (within a nearest-neighbour model), we find that both decrease smoothly as coordination is reduced. The effect of dimensionality appears to be small. We find that the force constants at equilibrium vary as the inverse eighth power of the equilibrium bond length. We also find evidence that the force constants are sensitive only to the bond length, and not to the coordination number. We believe these results will be useful in formulating interatomic potentials, e.g., for nanosystems.
\end{abstract}

Keywords. Coordination number; bond length; force constant; binding curve; density functional theory.

\section{Introduction}

With the recent interest in nanosystems, it is of interest to know how structures and properties change when coordination number is reduced. Such quantities can be calculated accurately using $a b$ initio density functional theory (DFT) methods. However, these computations can be extremely expensive (in terms of CPU time and memory requirements), especially if the loss of periodicity in one or more dimensions necessitates the use of large supercells that incorporate a vacuum region in the non-periodic dimensions. For those systems that have large unit cells that are not accessible to ab initio computation, an alternative approach would be to use semi-empirical potentials or force fields that can accurately reproduce the effects of reduced coordination, both qualitatively and quantitatively.

Many such potentials and approaches have been developed in recent times, e.g. using effective medium theory (Jacobsen et al 1987) and the embedded atom method (Foiles et al 1986). Typically, these approaches consist of a pair potential and a many-electron term dependent on the ambient electron density from neighbouring atoms. The parameters that specify these interactions are determined by fitting to a database consisting of experimental data and/or results of $a b$ initio calculations. One problem that arises is that such potentials become unreliable when applied to situations where the coordination number differs significantly from that in the systems to which the parameters were fitted. For example, a potential that was fit to bulk data cannot be guaranteed to reproduce surface properties accurately, and may be expected to be even more inaccurate when applied to systems such as nanowires and clusters.

*Author for correspondence (shobhana@jncasr.ac.in)
In this paper, we investigate whether it is possible to get some guidance in developing interatomic potentials by examining a large number of small-unit-cell periodic systems with varying coordination numbers. In a Taylor series expansion of pairwise interatomic potentials, the leading terms involve $l_{0}$, the position of the minimum, and $k_{0}$, which is related to the second derivative of the energy, evaluated at $l_{0}$. We wish to investigate how $l_{0}$ and $k_{0}$ vary with the coordination number, $\mathrm{CN}$. In particular, we are interested in examining whether there are universal scaling relations, which could then be used as a guide when developing interatomic potentials.

\section{Methods}

We have investigated a variety of hypothetical periodic structures spanning a range of coordination numbers for a number of elemental substances. In particular, we have considered the following three-dimensional (3D) structures: face-centred-cubic $(f c c)$, body-centred-cubic $(b c c)$, simple cubic $(s c)$, and diamond structure, which have $\mathrm{CN}=12,8,6$ and 4 , respectively. In two-dimensions (2D), we have considered the triangular lattice $(\mathrm{CN}=6)$, the square lattice $(\mathrm{CN}=4)$ and the honeycomb lattice $(\mathrm{CN}=3)$. Finally, in one-dimension (1D), we have considered the linear chain $(\mathrm{CN}=2)$. Note that all these structures are isotropic in all their periodic dimensions, and that their nearest-neighbour (NN) lengths are significantly shorter than their next-nearest-neighbour lengths. The elements that we have chosen to study are Al, Si, Sn, $\mathrm{Pb}$ and $\mathrm{Au}$. Note also that, in the form that these elements occur in nature, both the structure and the electronic properties differ.

We have investigated the structural dependence of the total energy of these periodic structures by making use of $a b$ initio density functional theory (Hohenberg and Kohn 
1964). Our calculations have been performed using the PWscf code which forms a part of the QuantumESPRESSO distribution, wherein the Kohn-Sham (1965) equations are solved making use of a plane wave basis set and pseudopotentials. For all elements we have used a plane-wave cutoff of $272 \mathrm{eV}$; we used norm-conserving pseudopotentials (Bachelet et al 1982) for all elements except $\mathrm{Au}$, for which we used an ultrasoft pseudopotential (Vanderbilt 1990). Exchange and correlation were treated with the local density approximation (LDA) according to the scheme of Perdew and Zunger (1981). Brillouin zone integrations were performed making use of k-points generated using the Monkhorst-Pack (1976) scheme. The number of k-points varied with the structure studied; it was verified that the results were converged with respect to the number of $\mathbf{k}$-points used. Convergence was aided by making use of the Methfessel-Paxton (1989) smearing technique. For all the 1D and 2D periodic structures, a vacuum region of $8 \AA$ was introduced in the non-periodic dimensions to prevent interaction between periodic images.

The method used was as follows: for each element, for each periodic structure, we obtained a curve of energy versus lattice constant. In figure 1, we have shown the results for $\mathrm{Au}$ as an example. By a simple scaling, these graphs were then converted to graphs of $\varepsilon$ (the total energy per NN bond) versus $x$, the length of NN bonds. (To do this, we made the implicit assumption that upon changing the crystal from its equilibrium bond length, the change in energy can be entirely attributed to the energy cost of changing NN bond lengths). Each of these curves was then fit to a Morse potential of the form

$$
\varepsilon(x)=A_{0}-2 A_{1} \exp \left(-A_{2}\left(x-l_{0}\right)\right)+A_{1} \exp \left(-2 A_{2}\left(x-l_{0}\right)\right),
$$

where $\varepsilon(x)$ represents the total energy per $\mathrm{NN}$ bond of length, $x, A_{0}$ is related to the energy of an isolated atom, $A_{1}$ the cohesive energy per $\mathrm{NN}$ bond, and $l_{0}$ the equilibrium

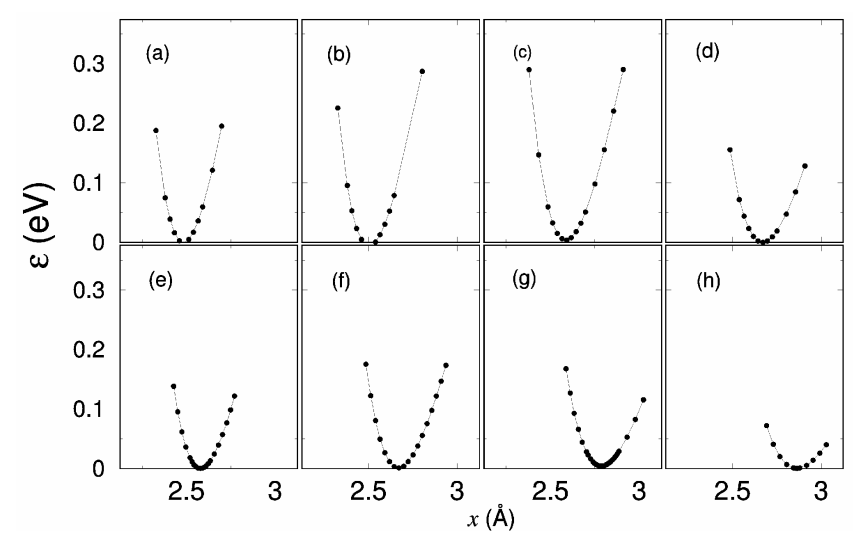

Figure 1. Ab initio results for $\varepsilon$ the total energy per NN bond as a function of bond length, $x$, for (a) linear chain, (b) honeycomb, (c) square, (d) triangular, (e) diamond, (f) simple cubic, (g) $b c c$ and (h) $f c c$ lattices, for $\mathrm{Au}$.
NN bond length for the given structure. The parameter, $A_{2}$, specifies the curvature of $\varepsilon(x)$ at its minimum; more precisely, the radial force constant per $\mathrm{NN}$ bond, at equilibrium, is (within our $\mathrm{NN}$ approximation) given by $k_{0}=2 A_{1} A_{2}^{2}$.

\section{Results and discussion}

In figure 2, we have shown how the equilibrium $\mathrm{NN}$ length, $l_{0}$, varies with coordination number $\mathrm{CN}$, for all the elements that we have considered. From these curves, we see that $l_{0}$ decreases as $\mathrm{CN}$ is reduced, regardless of the dimensionality of the system. While this behaviour is expected, based on the bond order-bond length arguments familiar to chemists, it is not clear, a priori, what the quantitative magnitude of this effect will be, and what role dimensionality plays. We find that the effect of dimensionality is small: in all cases, the values of $l_{0}$ vary by $<2 \%$ when we compare structures that have the same value of $\mathrm{CN}$ but different dimensions (e.g. the $2 \mathrm{D}$ square lattice and the $3 \mathrm{D}$ diamond lattice, or the $2 \mathrm{D}$ triangular lattice and the $3 \mathrm{D}$ simple cubic lattice). Compared to the overall variation of bond lengths by about $20-25 \%$ over the range of $\mathrm{CNs}$ we have considered, these differences are small. It is also interesting to note that in the case of the noble metal $\mathrm{Au}$, dimensionality appears to play almost no role, i.e. $l_{0}$ is essentially the same for the square and diamond lattices, and for the triangular and sc lattices. We also observe that with minor exceptions, the equilibrium bond lengths of 3D lattice systems are very slightly shorter than those of the 2D lattice systems with the same CN.

The variation of the equilibrium bond force constant, $k_{0}$, as a function of $\mathrm{CN}$ is plotted in figure 3. The data for

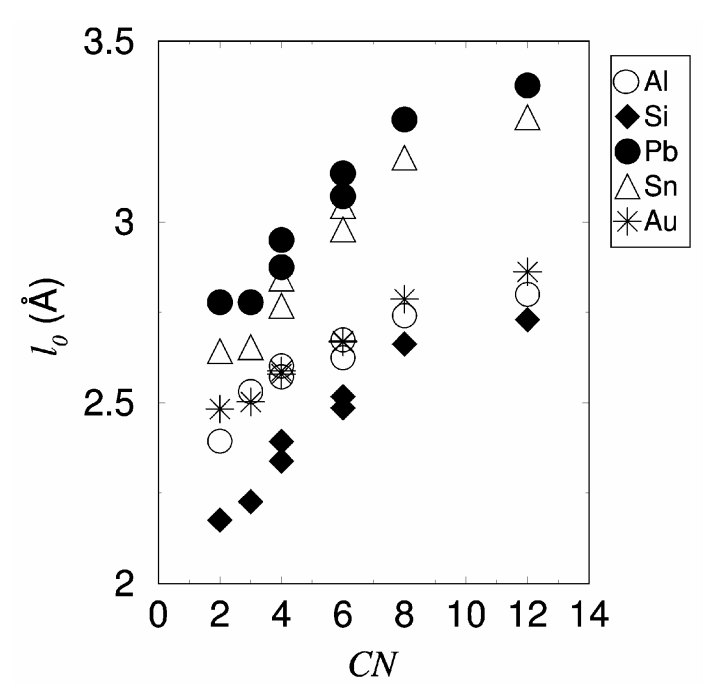

Figure 2. Equilibrium bond length, $l_{0}$, as a function of coordination number, $\mathrm{CN}$, for elements $\mathrm{Al}, \mathrm{Si}, \mathrm{Pb}, \mathrm{Sn}$ and $\mathrm{Au}$. Note that as $\mathrm{CN}$ decreases, the bonds become shorter. 
all the elements clearly show that as $\mathrm{CN}$ decreases, bonds become stiffer and the effects of dimensionality are small. In general, the $3 \mathrm{D}$ systems have stiffer bonds compared to $2 \mathrm{D}$ systems with the same $\mathrm{CN}$ except for $\mathrm{Al}$ and $\mathrm{Au}$ where $2 \mathrm{D}$ square and $3 \mathrm{D}$ diamond have nearly equal force constants. The differences in $k_{0}$ appear to be more noticeable than those in $l_{0}$. We found that even with very high k-point sampling, the results for the curvature of $\varepsilon(x)$ for the Si linear chain did not converge. It is possible that this is related to the Peierls' instability (Peierls 1955) in the linear chain; because of this we have not presented results for the Si linear chain.

In figure 4 , we have displayed the equilibrium bond force constant, $k_{0}$, as a function of equilibrium bond length, $l_{0}$. It is notable that these plots present much smoother trends than those obtained in figures 2 and 3. For all elements, with decreasing bond length, the force constant is seen to increase. We find that the set of points corresponding to each element can be adequately fitted with the functional form, $k_{0}=C / l_{0}^{8}$, where $C$ is a constant that depends on the element. This suggests that for a given element, $l_{0}, k_{0}$ can be obtained for any $\mathrm{CN}$, if the value of $l_{0}$ is known at any one value of $\mathrm{CN}$. This is an interesting result since with this simple model, the equilibrium bond length and equilibrium bond stiffness for any element can be obtained without performing a detailed set of calculations. We note that such a variation of force constants with bond lengths has also been observed in experiments

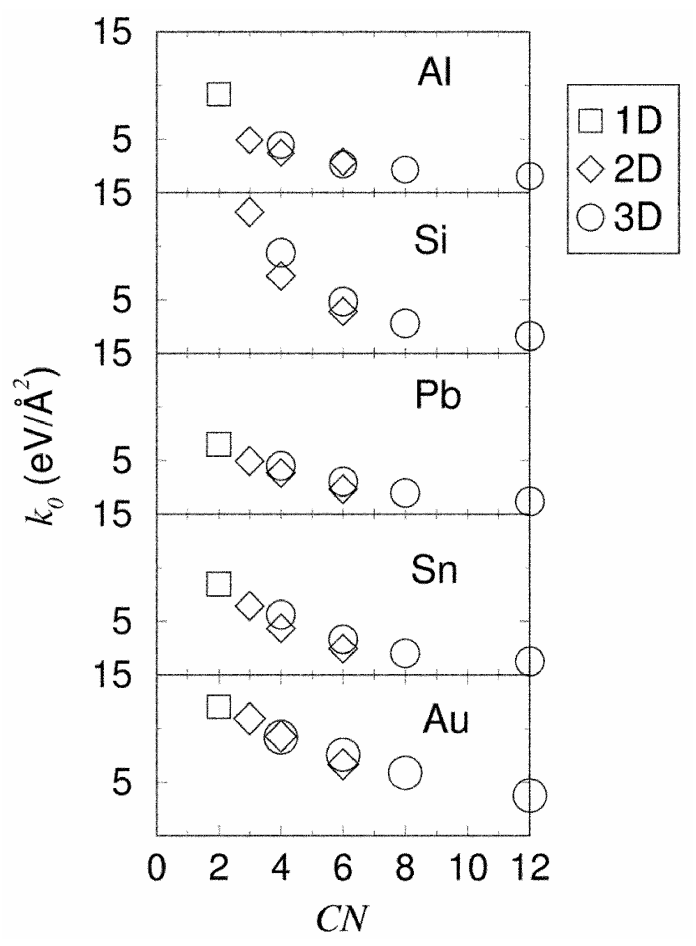

Figure 3. Equilibrium bond stiffness constant, $k_{0}$, as a function of coordination number, $\mathrm{CN}$, for elements $\mathrm{Al}, \mathrm{Si}, \mathrm{Pb}, \mathrm{Sn}$, and $\mathrm{Au}$. Note that as CN decreases, the bonds become stiffer. on surface vibrations (Zeppenfeld 1989); however, in the experiments the bond lengths varied due to changes in temperature or chemistry, whereas in our case they vary due to changes in $\mathrm{CN}$.

Only for the element $\mathrm{Au}$, we have also studied the effects on the force constants if all the structures are forced to have the $f_{c c}$ bond length as the NN bond length. Figure 5 shows how the force constants vary with $\mathrm{CN}$, both at equilibrium as well as at the $f c c$ bond length. It is striking that the former varies strongly with $\mathrm{CN}$, whereas the latter shows little variation with $\mathrm{CN}$. In other words, the force constants increase with decreasing $\mathrm{CN}$ only if the bond lengths are allowed to relax. This is of relevance to cases, e.g. surface bonds, where the bond lengths may be constrained even though the $\mathrm{CN}$ is reduced.

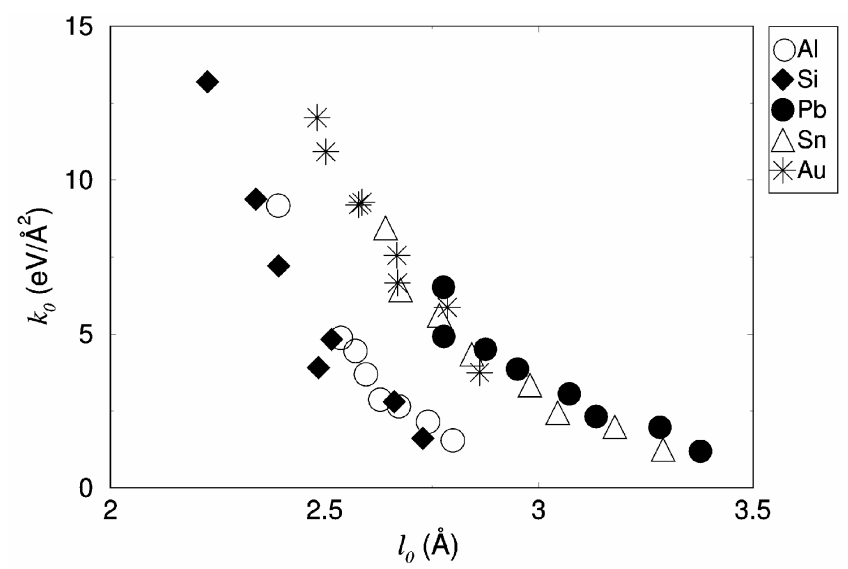

Figure 4. Equilibrium bond stiffness constant, $k_{0}$, as a function of equilibrium bond length, $l_{0}$, for all elements $\mathrm{Al}, \mathrm{Si}, \mathrm{Pb}$, $\mathrm{Sn}$ and $\mathrm{Au}$. The plots show a smooth trend in the variation of $k_{0}$ with respect to $l_{0}$

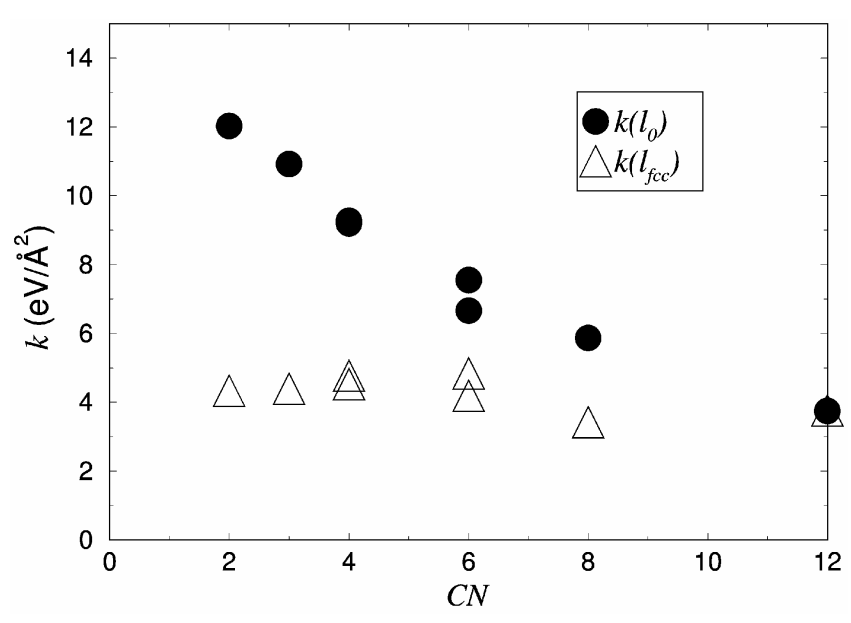

Figure 5. The variation of $k\left(l_{0}\right)$ and $k\left(l_{\mathrm{fcc}}\right)$, the radial force constants evaluated at the equilibrium bond length for $\mathrm{CN}$ and the bond length of the $f c c$ structure, respectively as a function of $\mathrm{CN}$. Note that $k\left(l_{f c c}\right)$ has little dependence on $\mathrm{CN}$, remaining approximately constant over the entire span of structures. 


\section{Conclusions}

Using DFT within LDA for exchange-correlation, we have studied the effects of reduced coordination on equilibrium bond lengths and force constants for $\mathrm{Al}, \mathrm{Si}, \mathrm{Pb}, \mathrm{Sn}$ and $\mathrm{Au}$. The coordination of each of these elements was varied by putting them in eight different structures. We have obtained smooth trends for $l_{0}$ and $k_{0}$ as functions of $\mathrm{CN}$ for each element. These trends show that with decreasing coordination, $l_{0}$ decreases and $k_{0}$ increases emphasizing the fact that shorter bonds correspond to stiffer bonds. These effects are only weakly affected by the dimensionality of the systems. For all the elements, the equilibrium force constants and bond lengths are related by the simple relation, $k_{0}=C / l_{0}^{8}$. One consequence of this relation is that, at equilibrium, the effects of dimensionality are larger on force constants than on bond lengths. Moreover, the increase in stiffness at lower coordinations occurs only if bond lengths can freely contract.

The kinds of relationships we have found between $k_{0}, l_{0}$ and $\mathrm{CN}$ can be used as a guide in developing interatomic potentials which can then be used to determine the properties of nanosystems.

\section{References}

Bachelet G B, Hamann D R and Schlüter M 1982 Phys. Rev. B26 4199

Foiles S M, Baskes M I and Daw M S 1986 Phys. Rev. B33 7983

Hohenberg P and Kohn W 1964 Phys. Rev. 136 B864

Jacobsen K W, Norskov J K and Puska M J 1987 Phys. Rev. B35 7423

Kohn W and Sham L J 1965 Phys. Rev. 140 A1133

Methfessel M and Paxton A T 1989 Phys. Rev. B40 3616

Monkhorst H J and Pack J D 1976 Phys. Rev. B13 5188

Peierls R 1955 Quantum theory of solids (Oxford, England: Oxford Univ. Press)

Perdew J P and Zunger A 1981 Phys. Rev. B23 5048

Quantum ESPRESSO: http://www.pwscf.org

Vanderbilt D 1990 Phys. Rev. B41 7892

Zeppenfeld D 1989 Ph.D. Thesis, KFA Jülich 